3 Koch-Weser J, Klein SW. Procainamide dosage schedules, plasma concentrations, and clinical effects. FAMA 1971;215:1454-60

4 Fontiverus ES, Cumming WJK, Hudson P. Procainamide induced myocitis. $\mathcal{f}$ Neurol Sci $1980 ; 45: 143-7$

5 Smith GT, Beeuwkes R, Tomkiewicz ZM, Tadarki A, Lown B. Pathological changes in skin and skeletal muscle following alternating current and capacitor discharge. Am f Pathol 1965;47:1-17.

(Accepted 5 December 1985)

Cardiac Department, King's College Hospital, London SE5 9RS

CAROLYN A LEWIS, MB, MRCP, medical registrar

N BOHEIMER, MB, FFARCS, anaesthetic registrar

P ROSE, MB, MRCPATH, lecturer in neuropathology

GRAHAM JACKSON, FRCP, consultant cardiologist

Correspondence to: Dr Jackson.

\section{Twenty four hour ambulatory electrocardiography in patients with chronic atrial fibrillation}

Ambulatory electrocardiography is widely used to detect paroxysmal cardiac arrhythmias. ' Asymptomatic arrhythmias may be detected, but their clinical importance cannot be assessed without comparative findings in healthy people. " 'Little attention has been paid to ambulatory electrocardiography in patients with established atrial fibrillation, in whom unexplained symptoms may prompt a search for a superimposed arrhythmia.

\section{Patients, methods, and results}

Seventy patients with chronic atrial fibrillation were investigated using 24 hour ambulatory electrocardiography (Oxford Medilog or Reynolds Tracker recorder, Reynolds Pathfinder 2 analyser). None had symptoms suggesting additional arrhythmias. In all patients the atrial fibrillation was "controlled" in that a change in treatment was not considered to be necessary. Satisfactory recordings were obtained in 66 patients ( 24 men and 42 women aged 38-94 (mean 63) years). Forty four patients had mitral valve disease (12 had undergone valve replacement), and the remainder had various cardiac conditions. Suppressant drugs comprised digoxin $(62 \cdot 5-750 \mu \mathrm{g} /$ day) in 59 cases, $\beta$ blocking drugs in eight, and verapamil in one. Most patients took additional drugs such as diuretics and anticoagulants. Heart rate was recorded in the clinic and blood taken for assay of serum digoxin concentration.

The figure shows the frequency distribution of fastest and slowest heart rates, longest pauses, and ventricular arrhythmias. The fastest heart rate occurred during the daytime in all patients and the slowest at night in 63 of 66 . The difference between fastest and slowest rates in individual patients ranged from 35 to 141 beats/min (mean 86 ). Heart rate measured in the clinic correlated poorly with fastest heart rate $(r=0.51)$ and slowest heart rate $(r=0.37)$. Only 16 of 27 patients with ambulatory heart rates above 140 beats/min had heart rates in the clinic above 90 beats $/ \mathrm{min}$, and in seven of 23 with rates in the clinic above 90 beats/min ambulatory rates did not exceed 140 beats/min. Daytime pauses of more than $2 \cdot 0$ seconds occurred in 16 of the 66 patients. No patient had daytime pauses longer than $2 \cdot 8$ seconds.
In the 59 patients taking digoxin there was no significant relation between serum digoxin concentration and fastest heart rate, slowest heart rate, duration of pauses, or frequency of ventricular arrhythmias. Five patients with serum digoxin concentrations above $2.0 \mu \mathrm{g} / \mathrm{l}$ but without clinically suspected digitalis toxicity could not be identified on the basis of bradycardia, pauses, or ventricular arrhythmias. No patient reported symptoms related to the documented changes in heart rate and arrhythmias.

\section{Comment}

Our results show the extremes of heart rate, duration of pauses, and frequency of ventricular arrhythmias detected by ambulatory electrocardiography in patients with clinically controlled atrial fibrillation. Recordings from symptomatic patients should be considered in the light of these findings before they are used as the basis for introducing or changing treatment.

Nocturnal pauses of up to 4.0 seconds and daytime pauses of up to $2 \cdot 8$ seconds may not require cardiac pacing unless they coincide with symptoms. Pauses, nocturnal bradycardia, and ventricular arrhythmias were common in patients who had no clinical or biochemical evidence of digoxin toxicity, and they should not be used to determine digoxin dosage. Intuitive selection of the dose of digoxin, however, often fails to achieve desired serum digoxin concentrations. ${ }^{3}$ Resting heart rate is an unreliable indicator of both digoxin concentration and control of heart rate during exercise. ${ }^{45}$ Heart rate during exercise is a major determinant of symptoms such as breathlessness, but the best method of assessing control of heart rate on exercise is uncertain. Standardised exercise tests allow comparison of the heart rate responses to certain levels of exercise but take no account of the different exercise requirements of people during daily activities. Ambulatory electrocardiography can assess variation in heart rate during daily life, but the optimal extremes of heart rate may differ between patients. Nevertheless, this technique may be useful for comparing the control of heart rate by different drugs in individual patients with atrial fibrillation.

We thank Drs S C Jordan and J V Jones for allowing us to study patients under their care; Mr L M Clarke and staff for technical help; and the department of medical illustration for preparing the figure.

1 Petch M. Lessons from ambulatory electrocardiography. Br Med f 1985;291:617-9.

2 Bjerregaard P. Continuous ambulatory electrocardiography in healthy adult subjects over a 24-hour period. Copenhagen: Mohn Aps; 1983.

3 Dodds SM, Nicholson PW, Rodgers EM, Mawer GE, Kenyon WI. Digoxin prescribing: a evaluation of clinical judgement. Br Med f 1978;ii:668-9.

4 Chamberlain DA, White RJ, Howard MR, Smith TW. Plasma digoxin concentrations in patients with atrial fibrillation. BrMed f 1970;iii:429-32.

5 Redfors A. Digoxin dosage and ventricular rate at rest and exercise in patients with atria fibrillation. Acta Med Scand 1971;190:321-33.

(Accepted 2 December 1985)

Cardiology Department, Bristol Royal Infirmary, Bristol BS2 8HW

DAVID PITCHER, $M B, M R C P$, senior registrar

MARK PAPOUCHADO, MB, MRCP, registrar

MICHAEL A JAMES, MB, MRCP, registra

J RUSSELL REES, MD, FRCP, consultant physician

Correspondence to: Dr D Pitcher, County Hospital, Hereford HR1 2ER.

Heart rates, longest pauses, and ventricular arrhythmias during 24 hour electrocardiography in 66 patients with chronic atrial fibrillation (Solid areas in graph of ventricular arrhythmias indicate patients with tachycardia.) 